\title{
Comunicação
}

[Communication $]$

\section{Eritrograma de híbridos de surubim (Pseudoplatystoma reticulatum X $P$. coruscans) mantidos em diferentes densidades de estocagem}

\author{
[Erythrogram of hybrids surubins (Pseudoplatystoma coruscans X P. reticulatum) kept \\ at different stocking densities] \\ C.R. Labarrère, P.M.C. Faria, E.A. Teixeira, M.M. Melo*
}

Escola de Veterinária - Universidade Federal de Minas Gerais - Belo Horizonte, MG

Peixes do gênero Pseudoplatystoma são conhecidos por sua elevada taxa de crescimento e bom rendimento de carcaça, assim como por sua carne de sabor delicado e sem mio-espinhos. Muitos estudos têm sido desenvolvidos com o objetivo de determinar as melhores condições de cultivo desses animais. Nestas situações, o perfil sanguíneo pode ser uma ferramenta relevante, uma vez que é considerado um indicador de saúde nos peixes, auxiliando, desta forma, a determinar a influência do manejo sobre a homeostase desses animais, o que possibilita a detecção precoce de condições que estejam afetando o desempenho da produção. Diante deste contexto e frente às escassas informações a respeito dos parâmetros hematológicos do gênero Pseudoplatystoma, objetivou-se analisar o perfil sanguíneo de peixes híbridos Pseudoplatystoma ( $P$. reticulatum $\mathrm{X} P$. coruscans) submetidos a diferentes densidades de estocagem em sistema de recirculação de água, em duas distintas fases de produção.

O experimento foi realizado em sistema de recirculação de água instalado em LaboratórioEscola de Aquacultura. Este sistema foi composto de 20 tanques circulares com 400 litros de volume útil, cujo fluxo de água foi de 480 litros por hora. Cada um dos tanques recebeu aeração suplementar. Utilizaram-se resistência elétrica e termostato para o controle e a manutenção da temperatura da água entre 27 e $29^{\circ} \mathrm{C}$. O oxigênio dissolvido foi analisado por oxímetro (MO-900® - Instrutherm) e manteve-se acima de 4,2mg/L. Os valores de $\mathrm{pH}$ foram verificados com a utilização de pHmetro (Portable pH Meter RL060P® Russel) e ficaram entre 6,7 e 7,8. Amônia e nitrito foram medidos semanalmente, por meio de kit comercial (Alfakit) e os valores não ultrapassaram $0,25 \mathrm{mg} / \mathrm{L}$ e $0,30 \mathrm{mg} / \mathrm{L}$, respectivamente. $\mathrm{O}$ fotoperíodo empregado foi de 11 horas de luz.

A água, após passar pelos tanques, foi recolhida em sistema de escoamento comum e direcionada para tratamento composto por três etapas. $\mathrm{Na}$ primeira etapa, a água é conduzida por filtro mecânico de brita. Em seguida, a água é direcionada, por meio de bombeamento, para filtro pressurizado de areia - segunda etapa - e, por fim, passa por filtro biológico - terceira etapa. Posteriormente a esses tratamentos, a água é novamente enviada para os tanques de criação dos peixes.

Alevinos cachapinta $(P$. reticulatum $\mathrm{X} \quad P$. coruscans) com aproximadamente $11 \mathrm{~g}$ foram mantidos em laboratório de aquacultura. Utilizaram-se 780 peixes, 540 no primeiro experimento e 240 no segundo, de modo a atingir as biomassas desejadas nos tratamentos. As diferentes densidades de estocagem (tratamentos) foram baseadas no experimento de Turra et al. (2009). Este estudo consistiu de dois experimentos independentes, correspondendo a duas fases de produção definidas em função do peso final (experimento 1 e experimento 2 ). 
No experimento 1, foram utilizados 540 juvenis de cachapinta, com peso médio inicial de $147,6 \pm 2,1 \mathrm{~g}$. Os peixes permaneceram no experimento até que atingissem $400 \mathrm{~g}$. O período necessário para alcançar este peso foi de 67 dias. Os híbridos foram distribuídos em cinco diferentes tratamentos, sendo o primeiro com a menor e o último com a maior densidade de estocagem. Cada tratamento teve quatro repetições, totalizando 20 tanques. As cinco densidades de estocagem estudadas foram: 27,5 (A), 47,5 (B), 67,5 (C), 87,5 (D) e 107,5 (E) peixes $/ \mathrm{m}^{3}$ de água. Para obtenção dessas densidades, foram utilizados 11, 19, 27, 35 e 43 peixes por tanque, respectivamente. A biomassa encontrada no início do tratamento correspondeu a 1623,$1 ; 2776,4 ; 3981,6 ; 5228,7$ e $6336,3 \mathrm{~g}$, respectivamente.

No experimento 2, foram utilizados 240 cachapintas, com peso inicial de 400,0 $\pm 1,4 \mathrm{~g}$, os quais permaneceram no experimento até atingirem $1000 \mathrm{~g}$. O período experimental foi de 72 dias. Os peixes foram distribuídos em cinco diferentes tratamentos, sendo o primeiro com a menor e o último com a maior densidade de estocagem. Cada tratamento teve quatro repetições, assim como no experimento 1. As cinco densidades de estocagem estudadas foram: 10 (A), 20 (B), 30 (C), 40 (D) e 50 (E) peixes $/ \mathrm{m}^{3}$ de água. Para obtenção dessas densidades, foram utilizados quatro, oito, 12,16 e 20 peixes por tanque, respectivamente. A biomassa encontrada no início do tratamento correspondeu a 1596,2; 3197,2; 4804,3, 6401,0 e 8023,5g, respectivamente.

Para a coleta de sangue, foram capturados, aleatoriamente, dois peixes de cada caixa, dos experimentos 1 e 2 . Dessa forma, cada densidade estudada apresentou oito repetições, totalizando 40 animais em cada experimento.

Para a coleta de sangue, utilizou-se como protocolo anestésico a quinaldina (Merck), na concentração de $1 \mathrm{~mL} / 10 \mathrm{~L}$ de água. A coleta de sangue, em ambos os grupos, foi realizada após o período experimental, por punção da veia caudal, utilizando-se anticoagulante EDTA a $10 \%$. Foram analisadas as concentrações de hemoglobina $(\mathrm{Hg})$ por método colorimétrico em espectrofotômetro (espectrofotômetro Coleman) e kit comercial (Bioclin $囚)$; o volume globular (VG) pela técnica do micro-hematócrito (Micro Spin); e a concentração de hemoglobina globular média (CHCM) foi calculada segundo Thrall et al. (2006).

O delineamento experimental aplicado foi inteiramente ao acaso. Os resultados foram analisados pelos programas de computador Statistical Analysis System (SAS) e Sistema para Análises Estatísticas e Genéticas (SAEG). Foram realizados testes de normalidade (Lillifors) e homocedasticidade (Cochran e Bartlleti). As variáveis que apresentaram distribuição normal foram submetidas à análise de variância, e as comparações das médias foram feitas pelo teste de Tukey. O projeto de pesquisa foi aprovado pelo Comitê de Ética em Experimentação Animal da UFMG, com o número de protocolo $88 / 2008$.

No experimento 1, os valores médios de hemoglobina variaram de 7,06 (densidade E) a 7,93g/dL (densidade C), sem diferença estatística entre os grupos. Também não foi observada diferença $(\mathrm{P}>0,05)$ entre os valores médios de $\mathrm{VG}$, que ficaram entre 22,87 (densidade C) e 28,77\% (densidade D). Em relação ao CHCM, os valores médios das densidades $\mathrm{B}(32,78 \mathrm{~g} / \mathrm{dL})$ e $\mathrm{C}$ $(35,41 \mathrm{~g} / \mathrm{dL})$ diferem estatisticamente dos valores da densidade $\mathrm{D}(25,38 \mathrm{~g} / \mathrm{dL})$, mas foram semelhantes $(\mathrm{P}<0,05)$ às demais (Tab. 1).

No experimento 2, a concentração de hemoglobina variou de 7,31 g/dL (densidade E) a 8,11g/dL (densidade B). Em relação ao $\mathrm{VG}$, o menor e o maior valor foram 29,0 na densidade A e $32,3 \%$ na densidade C. A concentração de CHCM variou de $24,19 \mathrm{~g} / \mathrm{dL}$ na densidade C a $28,85 \mathrm{~g} / \mathrm{dL}$ na densidade A. Nenhuma característica hematológica avaliada neste experimento apresentou diferenças $(\mathrm{P}>0,05)$ entre grupos (Tab. 2). 


\section{Labarrère et al.}

Tabela 1. Valores médios e desvios-padrão de hemoglobina, volume globular (VG) e concentração de hemoglobina corpuscular média (CHCM) de híbridos Pseudoplatystoma sp., com peso médio final de $147,6 \pm 2,1 \mathrm{~g}$, cultivados em cinco densidades de estocagem (experimento 1)

\begin{tabular}{lcccccc} 
& Densidade A & Densidade B & Densidade C & Densidade D & Densidade E & CV \\
\hline Hemoglobina*,g/dL) & $7,59 \pm 1,53 \mathrm{~A}$ & $7,25 \pm 1,24 \mathrm{~A}$ & $7,93 \pm 3,33 \mathrm{~A}$ & $7,35 \pm 1,4 \mathrm{~A}$ & $7,06 \pm 0,98 \mathrm{~A}$ & 25,27 \\
$\mathrm{VG}^{*}, \%$ & $27,37 \pm 4,20 \mathrm{~A}$ & $23,12 \pm 5,69 \mathrm{~A}$ & $22,87 \pm 7,62 \mathrm{~A}$ & $28,77 \pm 4,11 \mathrm{~A}$ & $27,44 \pm 4,52 \mathrm{~A}$ & 20,54 \\
$\mathrm{CHCM}^{* *}, \mathrm{~g} / \mathrm{dL}$ & $27,88 \pm 4,24 \mathrm{AB}$ & $32,78 \pm 7,70 \mathrm{~B}$ & $35,41 \pm 11,61 \mathrm{~B}$ & $25,38 \pm 1,98 \mathrm{~A}$ & $26,11 \pm 3,7 \mathrm{AB}$ & 22,65 \\
\hline
\end{tabular}

Médias seguidas de letras distintas diferem entre os grupos (densidade de estocagem), pelos testes Tukey* e KruskalWallis** $(\mathrm{P}<0,05)$.

A: 27,5 peixes $/ \mathrm{m}^{3} ;$ B: 47,5 peixes $/ \mathrm{m}^{3}$; C: 67,5 peixes $/ \mathrm{m}^{3} ; \mathrm{d}: 87,5$ peixes $/ \mathrm{m}^{3} ;$ E: 107,5 peixes $/ \mathrm{m}^{3}$.

Tabela 2. Valores médios e desvios-padrão de hemoglobina volume globular (VG) e com concentração de hemoglobina corpuscular média (CHCM) de híbridos Pseudoplatystoma sp., peso médio final de 400,0 1,4g, cultivados em cinco densidades de estocagem (experimento 2)

\begin{tabular}{lcccccc} 
& Densidade A & Densidade B & Densidade C & Densidade D & Densidade E & CV \\
\hline Hemoglobina, g/dL & $7,56 \pm 1,62$ & $8,11 \pm 2,33$ & $7,77 \pm 0,98$ & $7,85 \pm 1,34$ & $7,31 \pm 2,09$ & 22,73 \\
VG, \% & $29,00 \pm 5,04$ & $30,50 \pm 1,92$ & $32,30 \pm 2,13$ & $29,87 \pm 3,27$ & $30,25 \pm 3,28$ & 10,93 \\
CHCM, g/dL & $28,85 \pm 7,12$ & $26,49 \pm 7,49$ & $24,19 \pm 3,87$ & $25,72 \pm 3,53$ & $24,78 \pm 8,15$ & 24,75 \\
\hline
\end{tabular}

Não houve diferença estatística, entre os grupos, pela análise de variância $(\mathrm{P}>0,05)$.

$\mathrm{CV}=$ coeficiente de variação $(\%)$.

A: 10 peixes $/ \mathrm{m}^{3}$; B: 20 peixes $/ \mathrm{m}^{3}$; C: 30 peixes $/ \mathrm{m}^{3}$; D: 40 peixes $/ \mathrm{m}^{3}$; E: 50 peixes $/ \mathrm{m}^{3}$.

Os valores médios de hemoglobina foram de 7,06 a $7,93 \mathrm{~g} / \mathrm{dL}$, no experimento 1 , e de 7,31 a $8,11 \mathrm{~g} / \mathrm{dL}$, no experimento 2 , situando-se dentro do intervalo de 5 a 10g/dL estabelecido por Weiss et al. (2010). Fagundes (2005) dosou a concentração de hemoglobina de peixes da espécie $P$. coruscans com pesos médios de 14,04, 24,94 e 44,78g, e observou valores de 10,50, 8,05 e $10,10 \mathrm{~g} / \mathrm{dL}$, respectivamente. Tavares Dias et al. (2009), ao estudarem peixes híbridos do gênero Pseudoplatystoma (P. fasciatum X P. coruscans), encontraram intervalo entre $5,2-6,2 \mathrm{~g} / \mathrm{dL}$ de hemoglobina.

Segundo Weiss et al. (2010), os valores de VG em peixes oscilam ente 20 e $45 \%$, e ao se avaliar esta variável, é importante considerar que peixes mais ativos podem apresentar maiores valores de VG, pois têm maior demanda de oxigênio. Valores altos de VG também podem ser consequência do estresse decorrente da captura ou de anestesia inadequada durante a coleta de sangue. Os resultados deste experimento estão dentro dos limites propostos por Weiss et al. (2010). Fagundes (2005) encontrou valores médios de 22,70, 19,20 e 23,5\% de VG em P. coruscans com peso médio de 14,04, 24,94 e 44,78g, respectivamente, enquanto Tavares Dias et al. (2009), ao trabalharem com peixes híbridos $(P$. fasciatum X $P$. coruscans) pesando entre 568,0 e $1350,0 \mathrm{~g}$, estabeleceram valores de VG entre 30 e $35 \%$.
Weiss et al. (2010) afirmaram que a faixa de normalidade para a concentração de CHCM é de 18,0 a $30,0 \mathrm{~g} / \mathrm{dL}$, geralmente menor que a de mamíferos devido ao espaço ocupado pelo núcleo nos eritrócitos. No presente trabalho, os valores médios de CHCM das densidades $\mathrm{B}$ e $\mathrm{C}$ apresentaram-se acima de $30 \mathrm{~g} / \mathrm{dL}$, maiores que os valores estipulados por Weiss et al. (2010). Tavares Dias et al. (2009) verificaram um intervalo de 16,8$18,8 \mathrm{~g} / \mathrm{dL}$ para CHCM de peixes híbridos ( $P$. fasciatum $\mathrm{x} P$. coruscans) pesando entre $568 \mathrm{e}$ $1350 \mathrm{~g}$.

A variável CHCM é um índice utilizado para avaliação do grau de anemia, e é calculada a partir da concentração de hemoglobina e do valor de VG. Respostas eritrocitárias relacionadas à anemia podem estar associadas à discreta diminuição de CHCM. Já o aumento dessa característica pode estar relacionado com hemólise ou lipemia da amostra (Thrall et al., 2006). Neste trabalho, as amostras não apresentaram estas alterações sanguíneas. Dessa forma, as concentrações mais altas de CHCM encontradas nas densidades B e C, do experimento 1, podem ser explicadas pela diminuição do VG nos mesmos grupos, 23,12 e $22,87 \%$, respectivamente.

Vários são os fatores que podem alterar os valores dos índices hematimétricos, especialmente estímulos estressantes. Entre estes, a densidade de 
estocagem pode aumentar a concentração sanguínea de catecolaminas, resultando em alterações nos parâmetros sanguíneos. Portanto, estes índices podem ser utilizados como rápida identificação da perda homeostática de um organismo frente a um agente estressor, seja ele relacionado a um patógeno, à nutrição ou ao manejo (Tavares Dias et al., 2009).

Elevadas concentrações sanguíneas de catecolaminas são capazes de induzir trocas iônicas pela membrana eritrocitária, resultando na maior entrada de água nestas células. Portanto, a tumefação das hemácias, juntamente com a hemoconcentração em decorrência das catecolaminas, resulta em aumento do valor de VG e diminuição de CHCM, já que a quantidade de hemoglobina permanece inalterada (Hemming, 1989). Da mesma forma, Houston et al. (1996) sugeriram que as alterações hematológicas durante respostas ao estresse indicariam hemoconcentração ou hemodiluição em razão dos distúrbios osmorregulatórios.

Nikinmaa et al. (1983) sugeriram que o aumento na concentração de hemoglobina e nos valores de VG durante a resposta ao estímulo estressante pode ser decorrente do aumento do consumo de oxigênio. Nessas situações, o aumento da hemoglobina estaria relacionado com a maior capacidade de transportar oxigênio pelo sangue, na tentativa de suprir o aumento da demanda energética. Fagundes (2005) observou que $P$. coruscans submetidos aos procedimentos de transporte e exposição à luz apresentaram aumento do $\mathrm{VG}$, sugerido pelo pesquisador como tentativa do organismo de buscar maior aporte de oxigênio.

Outros trabalhos têm discutido as respostas hematológicas do organismo frente a agentes químicos, patógenos, manejo, transporte, alta densidade populacional e desnutrição (Gonçalves 2009; Tavares Dias et al., 2009; Weiss et al.,
2010). Segundo Ellis (1984), a intoxicação por nitrito também pode acarretar alterações hematológicas em peixes, pois o nitrito é facilmente absorvido pelas brânquias, alcançando rapidamente a corrente sanguínea e oxidando a hemoglobina, transformando-a em meta-hemoglobina. Em seguida, instala-se um quadro de anemia hemolítica à medida que os macrófagos esplênicos removem as hemácias anormais da circulação.

Tavares Dias et al. (2007) observaram diminuição do VG e aumento do $\mathrm{CHCM}$ em peixes híbridos (Piaractus mesopotamicus $\mathrm{x}$ Colossoma macropomum) infestados pelo crustáceo Dolops carvalhoi; Tavares Dias et al. (2009) relataram redução da concentração de hemoglobina e de VG, além de aumento de CHCM em híbridos Pseudoplatystoma sp. naturalmente infectados com Pseudomonas sp. em cultivo. A diminuição da concentração do VG pode ser decorrente de anemias ou redução da eritropoiese (Weiss et al., 2010).

Frischknecht et al. (1994) demonstraram que Oncorhynchus mykiss alimentados com dietas deficientes de vitamina $\mathrm{E}$ apresentaram diminuição $(\mathrm{P}<0,05)$ nos percentuais de $\mathrm{VG}$, e Camargo et al. (2005) verificaram aumento da concentração de hemoglobina e o VG em Rhamdia quelen conforme aumentava o nível proteico na dieta. Entretanto, Gonçalves (2009) não observou alterações nos índices hematimétricos de Colossoma macropomum tratados com dietas contendo probióticos.

Conclui-se que os peixes híbridos Pseudoplatystoma (Pseudoplatystoma reticulatum $\mathrm{X}$ Pseudoplatystoma coruscans) mantêm a homeostase sanguínea após diferentes condições de adensamento.

Palavras-chave: peixe, eritrograma, Pseudoplatystoma reticulatum, Pseudoplatystoma coruscans

\section{ABSTRACT}

The blood profile of hybrid fish was studied. Pseudoplatystoma (P. coruscans $X$ P. reticulatum) were submitted to different stocking densities in recirculating water system in two distinct phases of production. Hemoglobin concentration $(\mathrm{Hg})$, packed cell volume $(\mathrm{PCV})$ and mean corpuscular hemoglobin concentration (MCHC) were evaluated. There was no difference between the studied densities. Hemoglobin concentration, PCV and MCHC remained within the normal range for teleost fish.

Keywords: fish, erythrogram, Pseudoplatystoma reticulatum, Pseudoplatystoma coruscans 


\section{REFERÊNCIAS}

CAMARGO, S.O.; POUEY, J.L.; MARTINS, C. Parâmetros eritrocitários do jundiá (Rhamdia quelen) submetido à dieta com diferentes níveis de proteína. Cienc. Rural, v.35, p.1406-1411, 2005.

ELLIS, A.E. Bizarre forms of erytrocytes in a specime of plaice, Pleuronectes platessa. J. Fish Dis., v.7, p.411-414, 1984.

FAGUNDES, M.; URBINATI, E.C. Stress in pintado (Pseudoplatystoma corruscans) during farming procedures. Aquaculture, v.276, p.112119,2008

FRISCHKNECHT, H.H.; WAHLI, T.; MEIER, W. Comparison of pathological changes due to deficiency of vitamin $\mathrm{C}$, vitamin $\mathrm{E}$ and combinations of vitamins $\mathrm{C}$ and $\mathrm{E}$ in raibow trout, Oncorhynchus mykiss. J. Fish Dis., v.17, p.31-45, 1994.

GONÇALVES, A. Hematologia e macrófagos policariontes em Colossoma macropomum, mantidos em duas densidades de estocagem, alimentados com dieta contendo probiótico e espirulina. 2009. 65f. Tese (doutorado) - Centro de Aquacultura, Universidade Estadual Paulista, Jaboticabal, SP.

HEMING, T.A. Clinical studies of fish blood: importance of sample collection and mensurement techniques. Amer. J. Vet. Res., v.50, p.93-97, 1989.

HOUSTON. A.H.; ROBERTS. W.C.; KENNINGTON. J.A. Hematological response in fish: pronephric and splenic involvements in the goldfish. Carassius auratus L. Fish Physiol. Bioch., v.15, p.481-489, 1996.
NIKINMAA, M.; SOVIO. A.; NAKARI. T. et al. Handling stress in brown trout (Salmo truta): physiological responses to transport in fresh water, and recovery in natural brackish water. Aquaculture, v.34, p.93-99, 1983.

SAS. SAS/STAT software: changes and enhancements through release 6.12. Cary: Statistical Analysis System Institute, 1997. $1167 \mathrm{p}$.

TAVARES DIAS, M.; ISHIKAWA, M.M.; MARTINS, M.L. et al. Hematologia: ferramenta para o monitoramento do estado de saúde de peixes em cultivo. In: SARAN-NETO; MARIANO, W.S.; POZZOBON-SORIA. Tópicos Especiais em Saúde e Criação Animal. São Carlos: Pedro \& João Editores, 2009. cap. 3, 43-80 p.

TAVARES-DIAS, M.; MORAES, F.R.; ONAKA, E.M. et al. Changes in blood parameters of hybrid tambacu fish parasitized by Dolops carvalhoi (Crustacea, Branchiura), a fish louse. Vet. Arh., v.77, p.355-363, 2007.

THRALL, M.A; BAKER, D.C; CAMPBELL, T.W. et al. Hematologia e Bioquímica Clínica Veterinária. São Paulo: Rocca, 2006. 582p.

TURRA, E.M.; QUEIROZ, B.M.; TEIXEIRA, E.A. et al. Densidade de estocagem do surubim Pseudoplatystoma spp. cultivado em tanquerede. Rev. Bras. Saúde Prod. Anim., v.10, p.177187, 2009.

WEISS, D.J.; WARDROP, J.; SCHALM, O.W. Schalm's Veterinary Hematology. 6.ed. Iowa: Blackwell Publishing, 2010. 1206p. 